\title{
A Case of Needle-Directed Pulse-Spray Pharmacomechanical Thrombolysis of Acute Brachial Artery Thrombosis
}

\author{
Amitha Vikrama $\mathrm{KS}^{3}$ \\ ${ }^{1}$ Department of Radiology, Narayana Medical College and Hospital, \\ Nellore, Andhra Pradesh, India \\ 2Department of Radiology, Manipal Hospitals, Bangalore, \\ Karnataka, India \\ ${ }^{3}$ Department of Radiology, Sakra World Hospital, Bangalore, \\ Karnataka, India \\ J Clin Interv Radiol ISVIR 2019;3:193-195
}

Yugandhar Samireddypalle ${ }^{1, \odot ~ U m a ~ M a h e s h w a r a ~ R e d d y ~ V ~}{ }^{1} \quad$ Chinmay Deepak ${ }^{2}$ Sankar Neelakantan ${ }^{3}$

\begin{abstract}
Address for correspondence Yugandhar Samireddypalle, DNB, PDCC, Department of radiology, Narayana Medical College and Hospital, Nellore 524001, Andhra Pradesh, India

(e-mail: yugu.samireddypalle@gmail.com).
\end{abstract}
Abstract
Keywords
- thrombosis
- needle-directed pulse- spray pharmacome- chanical thrombolysis
- recanalization

Acute limb ischemia is a serious disabling condition. The management options depend upon the patient symptoms and signs of ischemia in the affected limb. The management options include surgical thrombo-embolectomy and catheter directed thrombolysis. Catheter directed thrombolysis has now become the first line of management in symptomatic cases. Here, we discuss a case of acute left brachial artery thrombosis which was successfully managed by needle-directed pulse-spray thrombolysis.

\section{Case Report}

A 55-year-old male patient was admitted to the hospital with complaints of acute severe left upper limb pain since 1 day. He has a heavy smoking history for more than 30 years and also history of intermittent claudication for which he underwent peripheral angioplasty 1 year back. On examination, his left upper limb was cold and clammy without any appreciable pulse. He complained of excruciating pain along the medial aspect of the forearm and the hand.

Doppler's examination was requested which revealed complete thrombosis of the brachial artery from the proximal upper arm ( - Fig. 1A, B) extending across the bifurcation into both proximal radial and ulnar arteries. The distal forearm arteries were collapsed. In view of the symptoms, he was advised catheter directed thrombolysis. As the patient had financial constraints, it was decided to offer him ultrasound guided needle-directed thrombolysis.

This procedure was done bedside. A thrombolytic solution was prepared by dissolving 5 lakh units of urokinase in $20 \mathrm{~mL}$ of heparinized saline (2,000U). Under all aseptic precautions, multiple 26 gauge needles were inserted into the left brachial artery

received

May 31, 2019

accepted

July 8, 2019

published online

December 4, 2019 at an equal distance of $5 \mathrm{~cm}$ and three-way stopcocks were connected ( - Fig. 2A). Under ultrasound guidance, $0.5 \mathrm{~mL}$ of this thrombolytic solution was injected forcefully into each of these needles with pressure ( $\mathbf{- F i g}$. 2B). Care was taken to exclude air bubble into the system. At the end of each session of injection, Doppler's examination was performed to look for restoration of flow. There was near total recanalization of the brachial artery within 30 minutes with mild residual clots ( - Fig. 3A, B). About 4 lakh units of urokinase were administered.

Postprocedure, the patient was kept on low-molecularweight heparin in addition to antiplatelets for a couple of days. The patient had complete resolution of pain postprocedure and was discharged in a stable condition. Followup Doppler after 1 week was normal.

\section{Discussion}

Acute limb ischemia is a serious and limb threatening condition and immediate active intervention is required if the patient has severe clinical symptoms. Surgical thromboembolectomy used to be the procedure of choice previously, but with the advances in intervention radiology, catheter
License terms

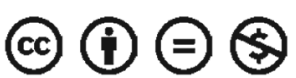




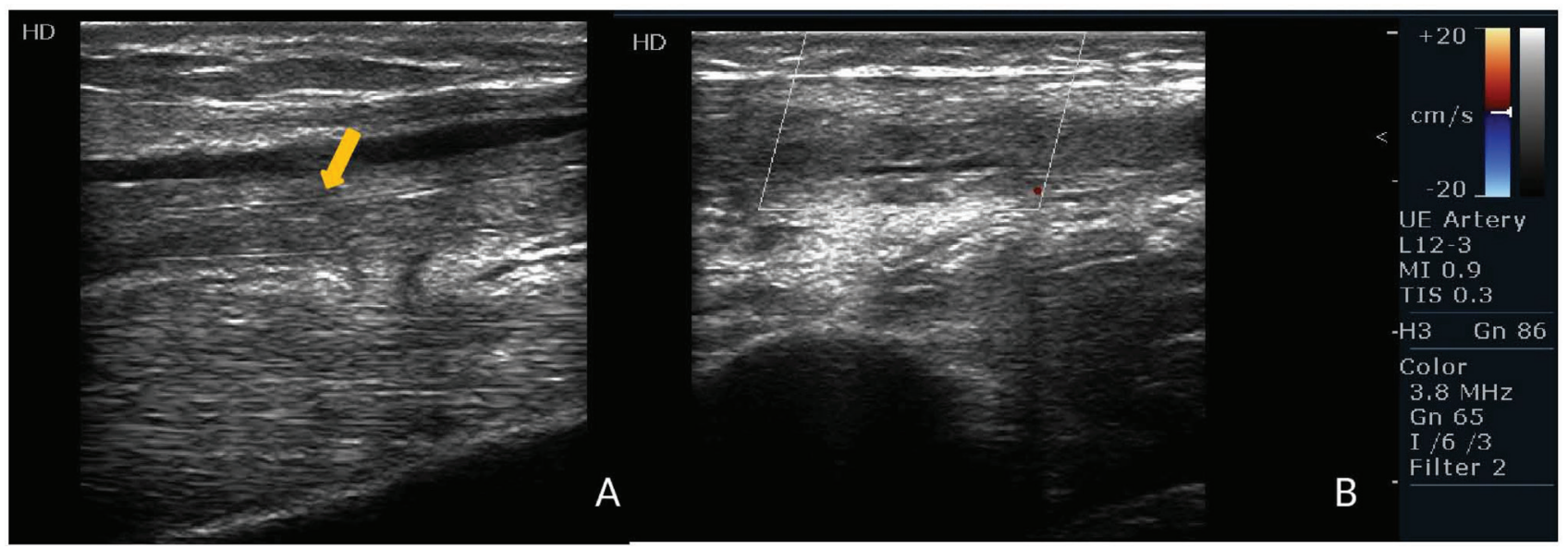

Fig. 1 (A) Ultrasound showing acute thrombosis of the brachial artery (yellow arrow). (B) No demonstration of flow on color Doppler.

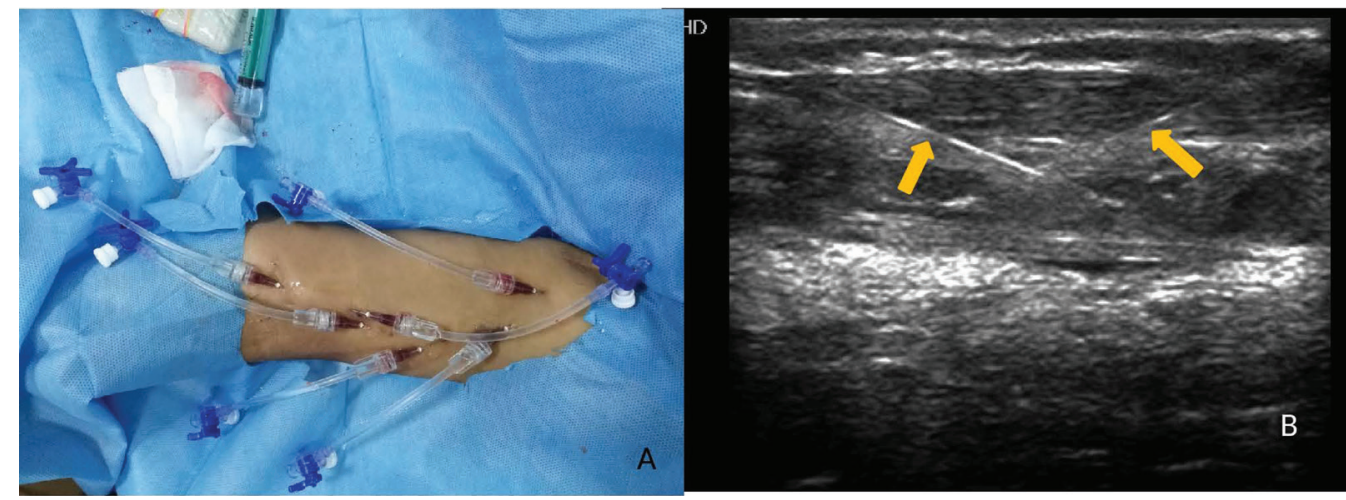

Fig. 2 (A) Image demonstrating the needle placements. (B) Two 26-gauge needles placed in criss-cross manner (yellow arrows) in the brachial artery at the proximal point of thrombus.

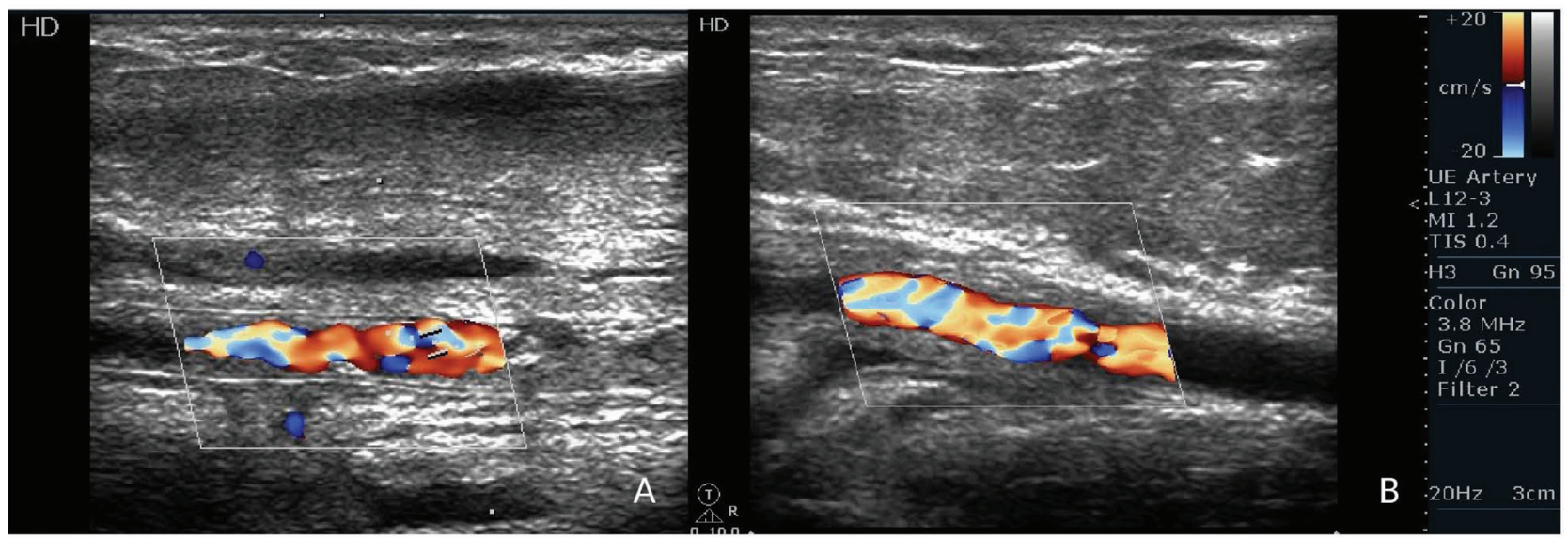

Fig. 3 (A) Color Doppler at the end of 30 minutes demonstrating near total recanalization with mild residual clots. (B) Follow-up Doppler after 24 hours demonstrating complete recanalization of the artery.

directed thrombolysis became the procedure of choice for treating these patients.

Catheter directed thrombolysis requires a cath-laboratory suite for positioning of infusion catheter. Prolonged infusion is usually required for several hours with intermittent Doppler or check angiograms. In addition, monitoring in high dependency unit (HDU) is mandatory during continuous thrombolytic infusion to monitor and detect any complication at the earliest. These procedures can be done in tertiary care centers with well-equipped laboratory and proper HDU care. Also, the cost factor for the intervention hardware and patient affordability has also to be considered. ${ }^{2}$

Needle-directed pulse-spray pharmacomechanical thrombolysis is a novel technique, which can be done bedside, under 
portable ultrasound guidance. ${ }^{3}$ It is an economical procedure as no intervention hardware is required. Each aliquot of injection can be monitored under ultrasound guidance and the procedure can be stopped at the earliest, once there is recanalization. We have performed needle-directed thrombolysis for more than 20 patients in our institution in salvaging acute thrombosis of dialysis arteriovenous grafts and fistulas.

This procedure requires proper intraluminal placement of needles at regular intervals. The proximal and distal needles have to be placed at the proximal and distal end of the thrombus as recanalization can be achieved only once there is both inflow and outflow. Each injection needs to be given forcefully for proper dissipation of the urokinase to achieve uniform thrombolysis. The procedure is stopped once there is optimal recanalization irrespective of clot burden. This is to prevent systemic infusion of urokinase. Low-molecular-weight heparin postprocedure will prevent rethrombosis, and it can be stopped once complete recanalization is achieved.

\section{Conclusion}

Needle-directed pulse-spray pharmacomechanical thrombolysis is an effective, economical, and easy technique for acute brachial artery thrombosis which can be done bedside under ultrasound guidance. However, larger studies are required to prove its safety and to compare its efficacy with catheter directed thrombolysis.

\section{Conflicts of Interest}

None declared.

\section{References}

1 Morrison HL. Catheter-directed thrombolysis for acute limb ischemia. Semin Intervent Radiol 2006; 23(3):258:269

2 Vikrama KA, Srivalli N, Venkataramana RS. USG-guided needle-directed pulse-spray pharmaco-mechanical thrombolysis of hemodialysis grafts/fistula: a novel technique. Indian J Radiol Imaging 2015;25(3):244-245

3 Vasanthraj PK, Bandi VK, Sai V, Shekar M. Pulse-spray pharmacomechanical thrombolysis of native arteriovenous fistulas and grafts under ultrasound guidance: our initial experience. Journal of Clinical Interventional Radiology ISVIR Vol. 3 No. 3/2019 2017;1(1):020-022 